\title{
Periodic Solutions for Second-Order Ordinary Differential Equations with Linear Nonlinearity
}

\author{
Xiaohong Hu, ${ }^{1}$ Dabin Wang, ${ }^{2}$ and Changyou Wang ${ }^{1}$ \\ ${ }^{1}$ School of Mathematics and Physics, Chongqing University of Posts and Telecommunications, Chongqing 400065, China \\ ${ }^{2}$ Department of Applied Mathematics, Lanzhou University of Technology, Lanzhou, Gansu 730050, China
}

Correspondence should be addressed to Xiaohong Hu; huxh@cqupt.edu.cn

Received 17 February 2013; Accepted 29 October 2013; Published 30 January 2014

Academic Editor: Pei Yu

Copyright (c) 2014 Xiaohong Hu et al. This is an open access article distributed under the Creative Commons Attribution License, which permits unrestricted use, distribution, and reproduction in any medium, provided the original work is properly cited.

By using minimax methods in critical point theory, we obtain the existence of periodic solutions for second-order ordinary differential equations with linear nonlinearity.

\section{Introduction and Main Results}

Consider the second-order ordinary differential systems

$$
\begin{aligned}
& \ddot{u}(t)+m^{2} \omega^{2} u(t)+\nabla F(t, u(t))=0, \quad \text { a.e. } t \in[0, T], \\
& u(0)-u(T)=\dot{u}(0)-\dot{u}(T)=0,
\end{aligned}
$$

where $T>0, \omega=2 \pi / T, m$ is a nonnegative integer; and $F:[0, T] \times R^{N} \rightarrow R$ satisfies the following assumption:

(A) $F(t, x)$ is measurable in $t$ for every $x \in R^{N}$ and continuously differentiable in $x$ for a.e. $t \in[0, T]$, and there exist $a \in C\left(R^{+}, R^{+}\right)$and $b \in L^{1}\left([0, T], R^{+}\right)$such that

$$
|F(t, x)| \leq a(|x|) b(t), \quad|\nabla F(t, x)| \leq a(|x|) b(t),
$$

for all $x \in R^{N}$ and a.e. $t \in[0, T]$, where $R^{+}$is the set of all nonnegative real numbers.

In the case of $m=0$, the existence of periodic solutions for problem (1) is obtained in articles [1-17] with many solvability conditions, such as the coercive type potential condition (see [1]), the convex type potential condition (see [2]), the periodic type potential conditions (see [3]), the even type potential condition (see [4]), the subquadratic potential condition in Rabinowitz's sense (see [5]), the bounded nonlinearity condition (see [6]), the subadditive condition (see [7]), the sublinear nonlinearity condition (see $[9,15])$, and the linear nonlinearity condition (see $[13,14,16,17])$.

In the case of $m \neq 0$, Mawhin and Willem [6] prove that problem (1) has at least one solution under the bounded nonlinearity condition; that is, $|\nabla F(t, x)| \leq g(t)$ for some $g \in L^{1}(0, T)$, each $x \in R^{N}$, and a.e. $t \in[0, T]$ when

$$
\begin{aligned}
& \int_{0}^{T} F(t, a \cos m \omega t+b \sin m \omega t) d t \\
& \quad \longrightarrow+\infty \text { as }|(a, b)| \longrightarrow \infty \text { in } R^{2 N}
\end{aligned}
$$

or

$$
\begin{aligned}
& \int_{0}^{T} F(t, a \cos m \omega t+b \sin m \omega t) d t \\
& \quad \longrightarrow-\infty \text { as }|(a, b)| \longrightarrow \infty \text { in } R^{2 N} .
\end{aligned}
$$

Under the sublinear nonlinearity condition, that is, there exist $f, g \in L^{2}[0, T]$ and $\alpha \in[0,1)$, such that

$$
|\nabla F(t, x)| \leq f(t)|x|^{\alpha}+g(t),
$$

for $x \in R^{N}$ and a.e. $t \in[0, T]$, Han [18] proves that problem (1) has at least one solution when

$$
\begin{gathered}
|(a, b)|^{-2 \alpha} \int_{0}^{T} F(t, a \cos m \omega t+b \sin m \omega t) d t \\
\longrightarrow+\infty \text { as }|(a, b)| \longrightarrow \infty \text { in } R^{2 N}
\end{gathered}
$$


or

$$
\begin{gathered}
|(a, b)|^{-2 \alpha} \int_{0}^{T} F(t, a \cos m \omega t+b \sin m \omega t) d t \\
\longrightarrow-\infty \text { as }|(a, b)| \longrightarrow \infty \text { in } R^{2 N} .
\end{gathered}
$$

Recently, when $m=0$, Zhao and $\mathrm{Wu}[13,14]$ and Meng and Tang $[16,17]$ also prove the existence of solutions for problem (1) under linear nonlinearity condition; that is, there exist $f, g \in L^{1}\left([0, T], R^{+}\right)$such that

$$
|\nabla F(t, x)| \leq f(t)|x|+g(t)
$$

In this paper, motivated by the results mentioned above, we investigate the existence of periodic solutions of problem (1) in the case of $m \geq 1$.

Let $H_{T}^{1}$ be a Hilbert space defined by

$$
\begin{gathered}
H_{T}^{1}=\left\{u:[0, T] \longrightarrow R^{N} \mid u\right. \text { is absolutely continuous, } \\
\left.u(0)=u(T) \text { and } \dot{u} \in L^{2}(0, T)\right\},
\end{gathered}
$$

with the norm

$$
\|u\|=\left(\int_{0}^{T}|u(t)|^{2} d t+\int_{0}^{T}|\dot{u}(t)|^{2} d t\right)^{1 / 2}
$$

for $u \in H_{T}^{1}$.

Let

$$
\begin{gathered}
H^{0}=\left\{a \cos m \omega t+b \sin m \omega t: a \in R^{N}, b \in R^{N}\right\}, \\
\bar{H}=\left\{\sum_{k=1}^{m-1} a_{k} \cos k \omega t+b_{k} \sin k \omega t: a_{k} \in R^{N}, b_{k} \in R^{N},\right. \\
1 \leq k \leq m-1\},
\end{gathered}
$$

$$
\begin{aligned}
\widetilde{H}=\left\{u \in H_{T}^{1}\right. & : \int_{0}^{T} u(t) \cos k \omega t d t \\
& \left.=\int_{0}^{T} u(t) \sin k \omega t d t=0,1 \leq k \leq m\right\} ;
\end{aligned}
$$

then $H_{T}^{1}=H^{0} \oplus \bar{H} \oplus \widetilde{H}([6])$. For all $u \in H_{T}^{1}$, we have $u=$ $u^{0}+\bar{u}+\widetilde{u}$, where $u^{0} \in H^{0}, \bar{u} \in \bar{H}$, and $\widetilde{u} \in \widetilde{H}$. It is easy to obtain

$$
\begin{aligned}
& \|\dot{\bar{u}}\|_{2}^{2} \leq(m-1)^{2} \omega^{2}\|\bar{u}\|_{2}^{2}, \quad \forall \bar{u} \in \bar{H}, \\
& \|\dot{\tilde{u}}\|_{2}^{2} \geq(m+1)^{2} \omega^{2}\|\widetilde{u}\|_{2}^{2}, \quad \forall \widetilde{u} \in \widetilde{H} .
\end{aligned}
$$

Furthermore, we have $\|u\|_{\infty} \leq C_{0}\|u\|$ for some $C_{0}>0$ and all $u(t) \in H_{T}^{1}$ (see, [6, Proposition 1.3]).

Our main results are the following theorems.
Theorem 1. Suppose that (A) and (8) hold and

(i)

$$
\begin{aligned}
(2+a) & C_{0}^{2} \int_{0}^{T} f(t) d t \\
& <\min \left\{\frac{(2 m+1) \omega^{2}}{1+(m+1)^{2} \omega^{2}}, \frac{(2 m-1) \omega^{2}}{1+(m-1)^{2} \omega^{2}}\right\},
\end{aligned}
$$

where $a$ is a parameter and satisfies $a>1 / 2$;

(ii)

$$
\begin{aligned}
\lim _{u \in H^{0},\|u\| \rightarrow \infty} & \inf \|u\|^{-2} \int_{0}^{T} F(t, u) d t \\
& >C_{0}^{2} \int_{0}^{T} f(t) d t+\frac{5 C_{0}^{2}}{2 a-1} \int_{0}^{T} f(t) d t+\frac{1}{2 a-1} .
\end{aligned}
$$

Then problem (1) has at least one solution.

Theorem 2. Suppose that (A), (8) and (i) hold and

$$
\begin{aligned}
\lim _{u \in H^{0},\|u\| \rightarrow \infty} & \sup \|u\|^{-2} \int_{0}^{T} F(t, u) d t \\
& <-\left[\frac{5 C_{0}^{2}}{2 a-1} \int_{0}^{T} f(t) d t+C_{0}^{2} \int_{0}^{T} f(t) d t+\frac{m^{2} \omega^{2}}{2 a-1}\right] .
\end{aligned}
$$

Then problem (1) has at least one solution.

Remark 3. (i) It is worth noting that, in the case of $m=0$, one solution was obtained by Tang [9] and Han [15] under the sublinear nonlinearity condition.

(ii) It is also worth noting that the sublinear nonlinearity condition in $[15,18]$ is different from that of $[9]$.

\section{Proof of Main Results}

Let

$$
\begin{aligned}
J(u)=\frac{1}{2} \int_{0}^{T}|\dot{u}(t)|^{2} d t-\frac{m^{2} \omega^{2}}{2} \\
\quad \times \int_{0}^{T}|u(t)|^{2} d t-\int_{0}^{T} F(t, u(t)) d t,
\end{aligned}
$$

for any $u \in H_{T}^{1}$. It follows from assumption $(A)$ that the functional $J$ on $H_{T}^{1}$ is continuously differentiable; moreover we obtain

$$
\begin{aligned}
\left\langle J^{\prime}(u), v\right\rangle= & \int_{0}^{T}(\dot{u}(t), \dot{v}(t)) d t-m^{2} \omega^{2} \\
& \times \int_{0}^{T}(u(t), v(t)) d t \\
& -\int_{0}^{T}(\nabla F(t, u(t)), v(t)) d t
\end{aligned}
$$


for any $u, v \in H_{T}^{1}$. It is well known that the solutions of problem (1) correspond to the critical points of $J$ (see [6]).

For the sake of convenience, we denote

$$
M_{1}=\int_{0}^{T} f(t) d t, \quad M_{2}=\int_{0}^{T} g(t) d t .
$$

Proof of Theorem 1. Firstly, we assert that the functional $J$ satisfies (PS) condition. Let $\left\{u_{n}\right\}$ be a sequence in $H_{T}^{1}$ such that $\left\{J\left(u_{n}\right)\right\}$ is bounded and $J^{\prime}\left(u_{n}\right) \rightarrow 0$ as $n \rightarrow \infty$. By the proof of [6] Proposition 4.1, we only need to prove that $\left\{u_{n}\right\}$ is bounded. On one hand, we have

\section{$\left\|\bar{u}_{n}\right\|$}

$$
\begin{aligned}
& \geq\left\langle J^{\prime}\left(\dot{u}_{n}\right),-\dot{\bar{u}}_{n}\right\rangle=-\int_{0}^{T}\left[\left(\dot{u}_{n}, \dot{\bar{u}}_{n}\right)-m^{2} \omega^{2}\left(u_{n}, \bar{u}_{n}\right)\right. \\
& \left.-\left(\nabla F\left(t, u_{n}\right), \bar{u}_{n}\right)\right] d t \\
& =-\int_{0}^{T}\left|\dot{\bar{u}}_{n}\right|^{2} d t+m^{2} \omega^{2} \\
& \times \int_{0}^{T}\left|\bar{u}_{n}\right|^{2} d t+\int_{0}^{T}\left(\nabla F\left(t, u_{n}\right), \bar{u}_{n}\right) d t \\
& \geq\left[m^{2}-(m-1)^{2}\right] \omega^{2} \\
& \times \int_{0}^{T}\left|\bar{u}_{n}\right|^{2} d t-\int_{0}^{T} f(t)\left|u_{n}^{0}+\bar{u}_{n}+\widetilde{u}_{n}\right|\left|\bar{u}_{n}\right| d t \\
& -\int_{0}^{T} g(t)\left|\bar{u}_{n}\right| d t \\
& \geq \frac{(2 m-1) \omega^{2}}{1+(m-1)^{2} \omega^{2}}\left\|\bar{u}_{n}\right\|^{2}-C_{0}^{2} M_{1}\left\|\bar{u}_{n}\right\|^{2} \\
& -C_{0}^{2} M_{1}\left\|\bar{u}_{n}\right\|\left\|\tilde{u}_{n}\right\|-C_{0}^{2} M_{1}\left\|\bar{u}_{n}\right\|\left\|u_{n}^{0}\right\|-C_{0} M_{2}\left\|\bar{u}_{n}\right\| \\
& \geq\left(\frac{(2 m-1) \omega^{2}}{1+(m-1)^{2} \omega^{2}}-2 C_{0}^{2} M_{1}\right) \\
& \times\left\|\bar{u}_{n}\right\|^{2}-\frac{C_{0}^{2} M_{1}}{2}\left\|\widetilde{u}_{n}\right\|^{2}-\frac{C_{0}^{2} M_{1}}{2}\left\|u_{n}^{0}\right\|^{2}-C_{0} M_{2}\left\|\bar{u}_{n}\right\| .
\end{aligned}
$$

So

$$
\begin{aligned}
& \frac{C_{0}^{2} M_{1}}{2}\left(\left\|\tilde{u}_{n}\right\|^{2}+\left\|u_{n}^{0}\right\|^{2}\right) \\
& \geq\left(\frac{(2 m-1) \omega^{2}}{1+(m-1)^{2} \omega^{2}}-(2+a) C_{0}^{2} M_{1}\right) \\
& \quad \times\left\|\bar{u}_{n}\right\|^{2}-\left(C_{0} M_{2}+1\right)\left\|\bar{u}_{n}\right\| \\
& \quad+a C_{0}^{2} M_{1}\left\|\bar{u}_{n}\right\|^{2} \\
& \geq a C_{0}^{2} M_{1}\left\|\bar{u}_{n}\right\|^{2}+C_{1},
\end{aligned}
$$

where $C_{1}=\min _{s \in[0, \infty)}\left\{\left(\left((2 m-1) \omega^{2} /\left(1+(m-1)^{2} \omega^{2}\right)\right)-(2+\right.\right.$ a) $\left.\left.C_{0}^{2} M_{1}\right) s^{2}-\left(C_{0} M_{2}+1\right) s\right\}$.
Since (14), so $-\infty<C_{1}<0$. Then

$$
\left\|\bar{u}_{n}\right\|^{2} \leq \frac{\left\|\tilde{u}_{n}\right\|^{2}}{2 a}+\frac{\left\|u_{n}^{0}\right\|^{2}}{2 a}+C_{2}
$$

where $C_{2}=-C_{1} / a C_{0}^{2} M_{1}>0$.

On the other hand, we have

$$
\begin{aligned}
\left\|\tilde{u}_{n}\right\| \geq & \left\langle J^{\prime}\left(u_{n}\right), \tilde{u}_{n}\right\rangle \\
\geq & \left(\frac{(2 m+1) \omega^{2}}{1+(m+1)^{2} \omega^{2}}-2 C_{0}^{2} M_{1}\right) \\
& \times\left\|\tilde{u}_{n}\right\|^{2}-\frac{C_{0}^{2} M_{1}}{2}\left\|\bar{u}_{n}\right\|^{2} \\
& -\frac{C_{0}^{2} M_{1}}{2}\left\|u_{n}^{0}\right\|^{2}-C_{0} M_{2}\left\|\tilde{u}_{n}\right\| .
\end{aligned}
$$

So

$$
\begin{aligned}
& \frac{C_{0}^{2} M_{1}}{2}\left(\left\|\bar{u}_{n}\right\|^{2}+\left\|u_{n}^{0}\right\|^{2}\right) \\
& \geq\left(\frac{(2 m+1) \omega^{2}}{1+(m+1)^{2} \omega^{2}}-(2+a) C_{0}^{2} M_{1}\right) \\
& \quad \times\left\|\tilde{u}_{n}\right\|^{2}-\left(C_{0} M_{2}+1\right)\left\|\tilde{u}_{n}\right\| \\
& \quad+a C_{0}^{2} M_{1}\left\|\tilde{u}_{n}\right\|^{2} \\
& \geq a C_{0}^{2} M_{1}\left\|\tilde{u}_{n}\right\|^{2}+C_{3},
\end{aligned}
$$

where $0>C_{3}=\min _{s \in[0, \infty)}\left\{\left(\left((2 m+1) \omega^{2} /\left(1+(m+1)^{2} \omega^{2}\right)\right)-\right.\right.$ $\left.\left.(2+a) C_{0}^{2} M_{1}\right) s^{2}-\left(C_{0} M_{2}+1\right) s\right\}$.

Then

$$
\left\|\tilde{u}_{n}\right\|^{2} \leq \frac{\left\|\bar{u}_{n}\right\|^{2}}{2 a}+\frac{\left\|u_{n}^{0}\right\|^{2}}{2 a}+C_{4},
$$

where $C_{4}=-C_{3} / a C_{0}^{2} M_{1}>0$.

From (22) and (25), we have

$$
\begin{aligned}
& \left\|\bar{u}_{n}\right\|^{2} \leq \frac{1}{2 a-1}\left\|u_{n}^{0}\right\|^{2}+C_{5}, \\
& \left\|\tilde{u}_{n}\right\|^{2} \leq \frac{1}{2 a-1}\left\|u_{n}^{0}\right\|^{2}+C_{5},
\end{aligned}
$$

where $C_{5}=\max \left\{\left(4 a^{2} C_{2}+2 a C_{4}\right) /\left(4 a^{2}-1\right),\left(4 a^{2} C_{4}+2 a C_{2}\right) /\right.$ $\left.\left(4 a^{2}-1\right)\right\}$. 
By (8), (26) we get

$$
\begin{aligned}
\left|\int_{0}^{T} F\left(t, u_{n}\right)-F\left(t, u_{n}^{0}\right) d t\right| \\
=\left|\int_{0}^{T} \int_{0}^{1} \nabla F\left(t, u_{n}^{0}+s\left(\bar{u}_{n}+\tilde{u}_{n}\right), u_{n}-u_{n}^{0}\right) d s d t\right| \\
\leq \int_{0}^{T} \int_{0}^{1} f(t)\left|u_{n}^{0}+s\left(\bar{u}_{n}+\tilde{u}_{n}\right)\right|\left|u_{n}-u_{n}^{0}\right| d t \\
\quad+\int_{0}^{T} \int_{0}^{1} g(t)\left|u_{n}-u_{n}^{0}\right| d t \\
\leq C_{0}^{2} M_{1}\left\|u_{n}^{0}\right\|^{2}+\frac{5}{2} C_{0}^{2} M_{1}\left\|\bar{u}_{n}\right\|^{2} \\
\quad+\frac{5}{2} C_{0}^{2} M_{1}\left\|\tilde{u}_{n}\right\|^{2}+C_{0} M_{2}\left(\left\|\bar{u}_{n}\right\|+\left\|\tilde{u}_{n}\right\|\right) \\
\leq \\
\quad\left(C_{0}^{2} M_{1}+\frac{5 C_{0}^{2} M_{1}}{2 a-1}\right)\left\|u_{n}^{0}\right\|^{2} \\
\quad+2 C_{0} M_{2} \sqrt{\frac{1}{2 a-1}}\left\|u_{n}^{0}\right\|+5 C_{5} C_{0}^{2} M_{1}+2 \sqrt{C_{5}} C_{0} M_{2} .
\end{aligned}
$$

It follows from (26), (27), and the boundedness of $J\left(u_{n}\right)$ that

$$
\begin{aligned}
J\left(u_{n}\right)= & \frac{1}{2} \int_{0}^{T}\left|\dot{u}_{n}\right|^{2} d t-\frac{m^{2} \omega^{2}}{2} \int_{0}^{T}\left|u_{n}\right|^{2} d t-\int_{0}^{T} F\left(t, u_{n}\right) d t \\
\leq & \frac{1}{2}\left(\left\|\bar{u}_{n}\right\|^{2}+\left\|\tilde{u}_{n}\right\|^{2}\right) \\
& -\int_{0}^{T} F\left(t, u_{n}\right)-F\left(t, u_{n}^{0}\right) d t-\int_{0}^{T} F\left(t, u_{n}^{0}\right) d t \\
\leq & \left(C_{0}^{2} M_{1}+\frac{5 C_{0}^{2} M_{1}}{2 a-1}+\frac{1}{2 a-1}\right) \\
& \times\left\|_{n}^{0}\right\|^{2}+2 C_{0} M_{2} \sqrt{\frac{1}{2 a-1}}\left\|u_{n}^{0}\right\| \\
& -\int_{0}^{T} F\left(t, u_{n}^{0}\right) d t+5 C_{5} C_{0}^{2} M_{1}+2 \sqrt{C_{5}} C_{0} M_{2} \\
= & \left\|u_{n}^{0}\right\|^{2}\left[C_{0}^{2} M_{1}+\frac{5 C_{0}^{2} M_{1}}{2 a-1}+\frac{1}{2 a-1}\right. \\
& +5 C_{5} C_{0}^{2} M_{1}+2 \sqrt{C_{5} C_{0} M_{2} .} \\
& \quad-\left\|u_{n}^{0}\right\|^{-2} \int_{0}^{T} M_{2} \sqrt{\frac{1}{2 a-1}}\left\|u_{n}^{0}\right\|^{-1}
\end{aligned}
$$

The above inequality and (15) imply that $\left\{u_{n}^{0}\right\}$ is bounded. Hence $\left\{u_{n}\right\}$ is bounded by (26).
Secondly, we assert that

$$
\begin{aligned}
& \left(J_{1}\right) J(u) \rightarrow+\infty \text { as }\|u\| \rightarrow \infty \text { in } \widetilde{H} \text {, which implies that } \\
& \quad \inf _{u \in \widetilde{H}} J(u)>-\infty \text {; } \\
& \left(J_{2}\right) J(u) \rightarrow-\infty \text { as }\|u\| \rightarrow \infty \text { in } H^{0} \oplus \bar{H},
\end{aligned}
$$

for all $u \in H^{0} \oplus \bar{H}$; that is, $u=u^{0}+\bar{u}$; then by (8) and (12) we have

$$
\begin{aligned}
& J(u)=\frac{1}{2} \int_{0}^{T}|\dot{u}(t)|^{2} d t-\frac{m^{2} \omega^{2}}{2} \int_{0}^{T}|u(t)|^{2} d t \\
& -\int_{0}^{T} F(t, u(t)) d t \\
& =\frac{1}{2}\left(\int_{0}^{T}|\dot{\bar{u}}(t)|^{2} d t-m^{2} \omega^{2} \int_{0}^{T}|\bar{u}(t)|^{2} d t\right) \\
& -\int_{0}^{T}\left[F\left(t, u^{0}+\bar{u}\right)-F\left(t, u^{0}\right)\right] d t-\int_{0}^{T} F\left(t, u^{0}\right) d t \\
& \leq \frac{1}{2}(1-2 m) \omega^{2}\|\bar{u}\|_{2}^{2} \\
& -\int_{0}^{T} \int_{0}^{1}\left(\nabla F\left(t, u^{0}+s \bar{u}\right), \bar{u}\right) d t-\int_{0}^{T} F\left(t, u^{0}\right) d t \\
& \leq \frac{1}{2}(1-2 m) \omega^{2}\|\bar{u}\|_{2}^{2}+\int_{0}^{T} f(t)|\bar{u}(t)|^{2} d t \\
& +\int_{0}^{T} f(t)|\bar{u}(t)|\left|u^{0}\right| d t \\
& +\int_{0}^{T} g(t)|\bar{u}(t)| d t-\int_{0}^{T} F\left(t, u^{0}\right) d t \\
& \leq \frac{1}{2}(1-2 m) \omega^{2}\|\bar{u}\|_{2}^{2}+C_{0}^{2} M_{1}\|\bar{u}\|^{2} \\
& +C_{0}^{2} M_{1}\left\|u^{0}\right\|\|\bar{u}\|+C_{0} M_{2}\|\bar{u}\|-\int_{0}^{T} F\left(t, u^{0}\right) d t \\
& \leq \frac{1}{2}(1-2 m) \omega^{2}\|\bar{u}\|_{2}^{2}+C_{0}^{2} M_{1}\|\bar{u}\|^{2} \\
& +\frac{C_{0}^{2} M_{1}}{2 a}\left\|u^{0}\right\|^{2}+\frac{a C_{0}^{2} M_{1}}{2}\|\bar{u}\|^{2} \\
& +C_{0} M_{2}\|\bar{u}\|-\int_{0}^{T} F\left(t, u^{0}\right) d t \\
& <\frac{1}{2}(1-2 m) \omega^{2}\|\bar{u}\|_{2}^{2} \\
& +\frac{(2+a) C_{0}^{2} M_{1}}{2}\left[1+(m-1)^{2} \omega^{2}\right]\|\bar{u}\|_{2}^{2} \\
& +C_{0} M_{2}[(m-1) \omega+1]\|\bar{u}\|_{2} \\
& +C_{0}^{2} M_{1}\left\|u^{0}\right\|^{2}-\int_{0}^{T} F\left(t, u^{0}\right) d t
\end{aligned}
$$




$$
\begin{aligned}
= & \left\{\frac{1}{2}(1-2 m) \omega^{2}+\frac{(2+a) C_{0}^{2} M_{1}}{2}\left[1+(m-1)^{2} \omega^{2}\right]\right\} \\
& \times\|\bar{u}\|_{2}^{2}+C_{0} M_{2}[(m-1) \omega+1]\|\bar{u}\|_{2} \\
& +\left\|u^{0}\right\|^{2}\left[C_{0}^{2} M_{1}-\left\|u^{0}\right\|^{-2} \int_{0}^{T} F\left(t, u^{0}\right) d t\right],
\end{aligned}
$$

for $\|u\| \rightarrow \infty$ in $X$ if and only if $\|\bar{u}\|_{2} \rightarrow \infty$ or $\left\|u^{0}\right\| \rightarrow$ $\infty$. So, by $m \geq 1,(14)$, and (15), we obtain $J(u) \rightarrow-\infty$ as $\|u\| \rightarrow \infty$ in $X$.

Let $u \in \widetilde{H}$; then by (8) and (13), we have

$$
\begin{aligned}
& J(u)=\frac{1}{2} \int_{0}^{T}|\dot{u}(t)|^{2} d t-\frac{m^{2} \omega^{2}}{2} \int_{0}^{T}|u(t)|^{2} d t \\
& -\int_{0}^{T} F(t, u(t)) d t \\
& \geq \frac{1}{2}\left(1-\frac{m^{2} \omega^{2}}{(m+1)^{2} \omega^{2}}\right) \int_{0}^{T}|\dot{\vec{u}}(t)|^{2} d t \\
& -\int_{0}^{T}[F(t, \tilde{u})-F(t, 0)] d t-\int_{0}^{T} F(t, 0) d t \\
& \geq \frac{1}{2} \frac{2 m+1}{(m+1)^{2}} \times \frac{(m+1)^{2} \omega^{2}}{1+(m+1)^{2} \omega^{2}}\|\tilde{u}\|^{2} \\
& -\int_{0}^{T} \int_{0}^{1}(\nabla F(t, s \tilde{u}), \tilde{u}) d t-\int_{0}^{T} F(t, 0) d t \\
& \geq \frac{1}{2} \frac{(2 m+1) \omega^{2}}{1+(m+1)^{2} \omega^{2}}\|\widetilde{u}\|^{2} \\
& -\int_{0}^{T} f(t)|\widetilde{u}|^{2} d t-\int_{0}^{T} g(t)|\widetilde{u}| d t-\int_{0}^{T} F(t, 0) d t \\
& \geq\left(\frac{1}{2} \frac{(2 m+1) \omega^{2}}{1+(m+1)^{2} \omega^{2}}-C_{0}^{2} M_{1}\right) \\
& \times\|\widetilde{u}\|^{2}-C_{0} M_{2}\|\tilde{u}\|-\int_{0}^{T} F(t, 0) d t .
\end{aligned}
$$

So, by (14), $J$ is bounded from below on $\widetilde{H}$.

Hence, by Rabinowitz's Saddle point Theorem (see [19, Theorem 4.6]), we obtain that the problem (1) has at least one solution.

Proof of Theorem 2. The proof of Theorem 2 is similar to the proof of Theorem 1, so we omit it here.

\section{Conflict of Interests}

The authors declare that there is no conflict of interests regarding the publication of this paper.

\section{Acknowledgments}

The authors thank Professor Zhang S. Q. for his guidance, Editor Pei Yu for his hard work, and the anonymous referees for their comments and suggestions. This paper is supported partially by NSF of China and by Dr. Start-up fund of Chongqing University of Posts and Telecommunications.

\section{References}

[1] M. S. Berger and M. Schechter, "On the solvability of semilinear gradient operator equations," Advances in Mathematics, vol. 25, no. 2, pp. 97-132, 1977.

[2] J. Mawhin, "Semicoercive monotone variational problems," Bulletin de la Classe des Sciences. Académie Royale de Belgique, vol. 73, no. 3-4, pp. 118-130, 1987.

[3] M. Willem, "Oscillations forces de systmes hamiltoniens," in Public. Smin. Analyse Non Linaire, Université de Besançon, 1981.

[4] Y. M. Long, "Nonlinear oscillations for classical Hamiltonian systems with bi-even subquadratic potentials," Nonlinear Analysis: Theory, Methods \& Applications, vol. 24, no. 12, pp. 16651671, 1995.

[5] P. H. Rabinowitz, "On subharmonic solutions of Hamiltonian systems," Communications on Pure and Applied Mathematics, vol. 33, no. 5, pp. 609-633, 1980.

[6] J. Mawhin and M. Willem, Critical Point Theory and Hamiltonian Systems, vol. 74 of Applied Mathematical Sciences, Springer, New York, NY, USA, 1989.

[7] C. Tang, "Periodic solutions of non-autonomous second order systems with $\gamma$-quasisubadditive potential," Journal of Mathematical Analysis and Applications, vol. 189, no. 3, pp. 671-675, 1995.

[8] C. Tang, "Periodic solutions of non-autonomous second order systems," Journal of Mathematical Analysis and Applications, vol. 202, no. 2, pp. 465-469, 1996.

[9] C.-L. Tang, "Periodic solutions for nonautonomous second order systems with sublinear nonlinearity," Proceedings of the American Mathematical Society, vol. 126, no. 11, pp. 3263-3270, 1998.

[10] C.-L. Tang and X.-P. Wu, "Periodic solutions for second order systems with not uniformly coercive potential," Journal of Mathematical Analysis and Applications, vol. 259, no. 2, pp. 386$397,2001$.

[11] X. Wu, "Saddle point characterization and multiplicity of periodic solutions of non-autonomous second-order systems," Nonlinear Analysis: Theory, Methods \& Applications, vol. 58, no. 7-8, pp. 899-907, 2004.

[12] X.-P. Wu and C.-L. Tang, "Periodic solutions of a class of nonautonomous second-order systems," Journal of Mathematical Analysis and Applications, vol. 236, no. 2, pp. 227-235, 1999.

[13] F. Zhao and X. Wu, "Periodic solutions for a class of nonautonomous second order systems," Journal of Mathematical Analysis and Applications, vol. 296, no. 2, pp. 422-434, 2004.

[14] F. Zhao and X. Wu, "Existence and multiplicity of periodic solution for non-autonomous second-order systems with linear nonlinearity," Nonlinear Analysis: Theory, Methods \& Applications, vol. 60, no. 2, pp. 325-335, 2005.

[15] Z. Q. Han, “2 $\pi$-periodic solutions to $n$-Duffng systems," in Nonlinear Analysis and Its Aplications, D. J. Guo, Ed., pp. 182-191, Beijng Scientific and Technical, Beijing, China, 1994, (Chinese). 
[16] Q. Meng and X. H. Tang, "Solutions of a second-order Hamiltonian system with periodic boundary conditions," Communications on Pure and Applied Analysis, vol. 9, no. 4, pp. 1053-1067, 2010.

[17] X. H. Tang and Q. Meng, "Solutions of a second-order Hamiltonian system with periodic boundary conditions," Nonlinear Analysis: Real World Applications, vol. 11, no. 5, pp. 3722-3733, 2010.

[18] Z. Q. Han, " $2 \pi$-periodic solutions to ordinary differential systems at resonance," Acta Mathematica Sinica. Chinese Series, vol. 43, no. 4, pp. 639-644, 2000 (Chinese).

[19] P. H. Rabinowitz, Minimax Methods in Critical Point Theory with Applications to Differential Equations, vol. 65 of CBMS Regional Conference Series in Mathematics, American Mathematical Society, Washington, DC, USA, 1986. 


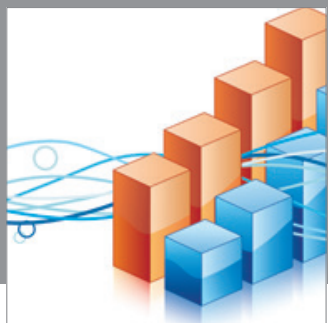

Advances in

Operations Research

mansans

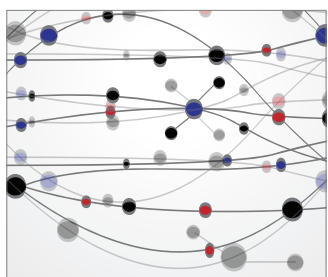

The Scientific World Journal
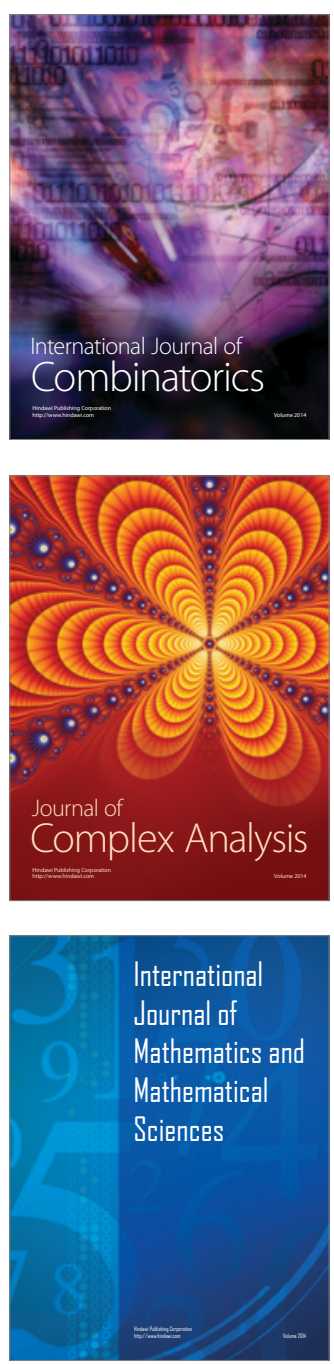
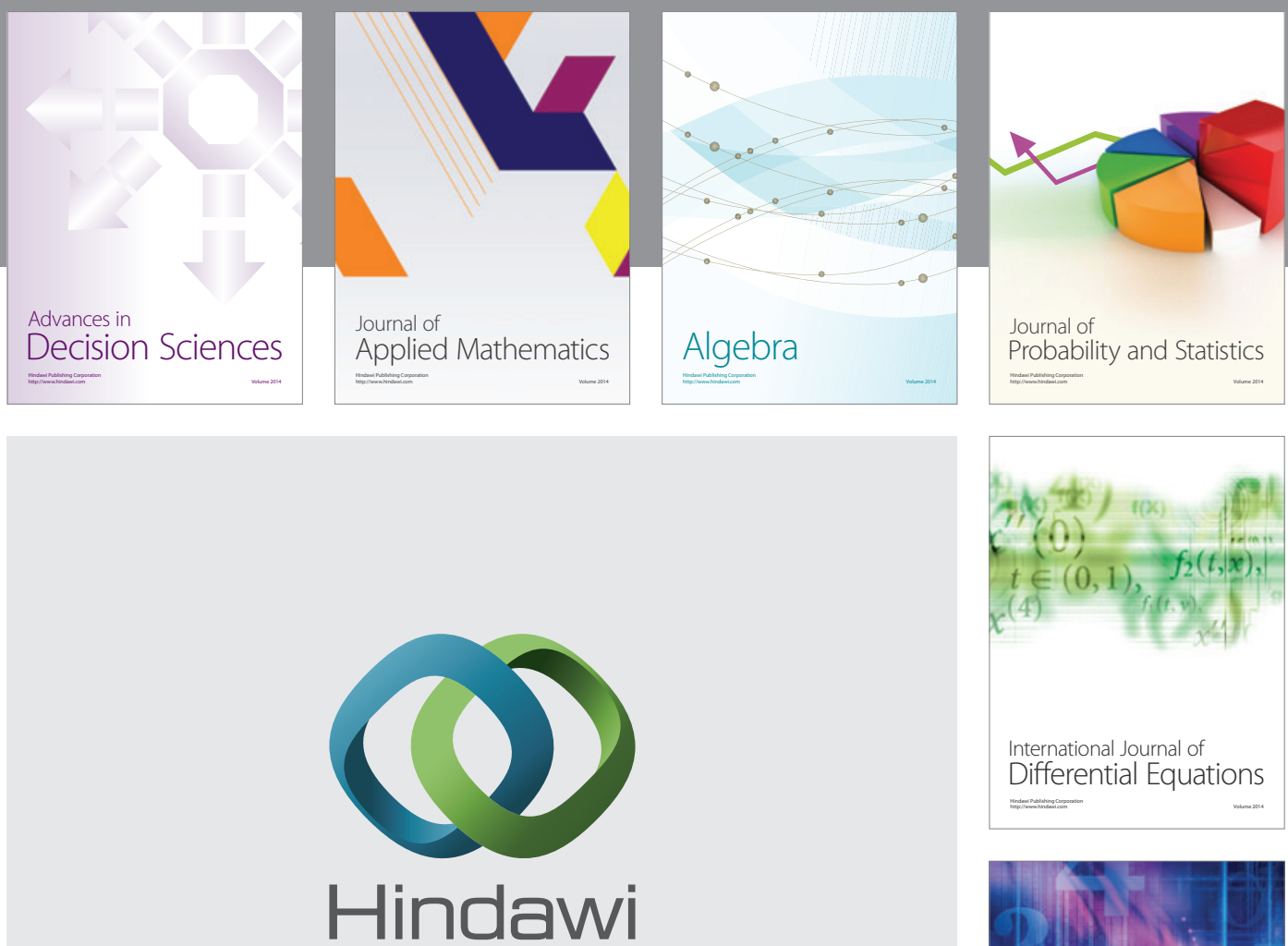

Submit your manuscripts at http://www.hindawi.com
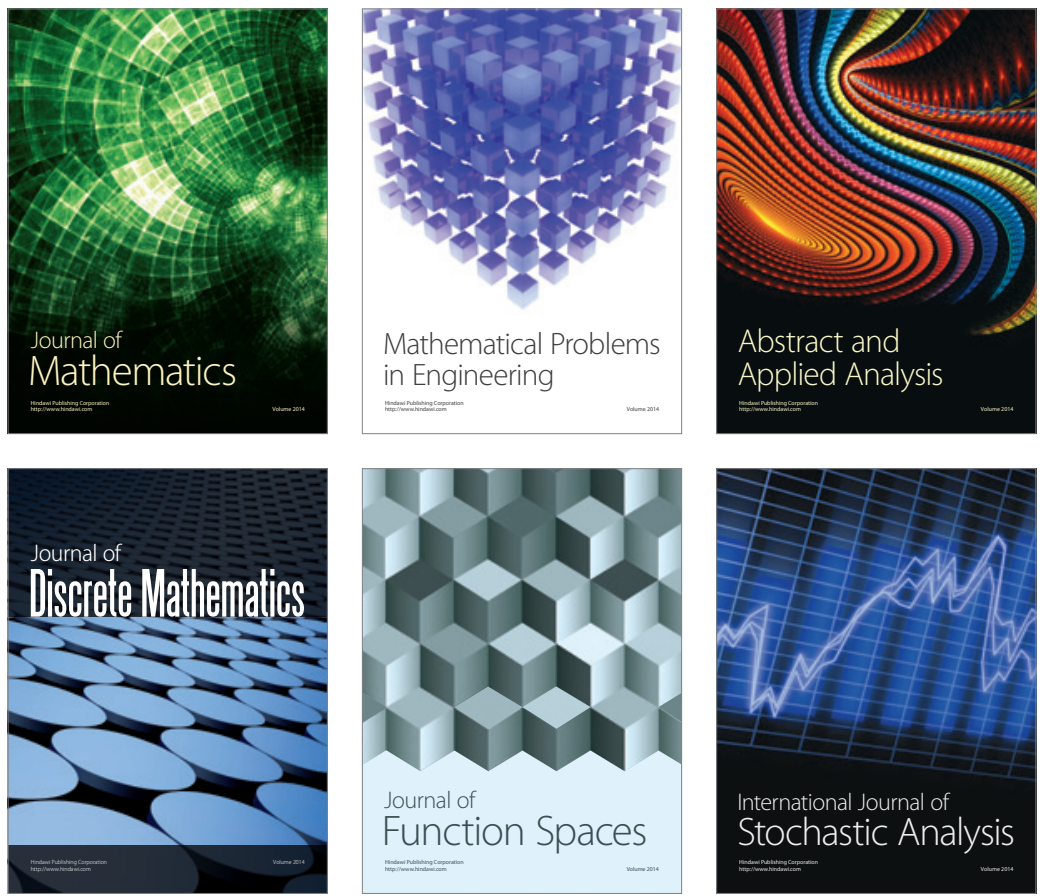

Journal of

Function Spaces

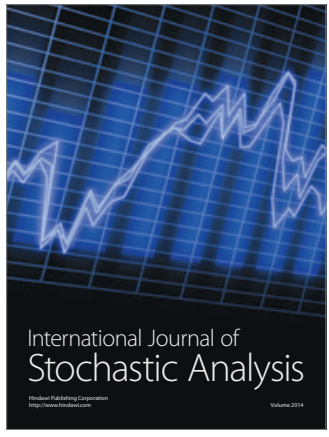

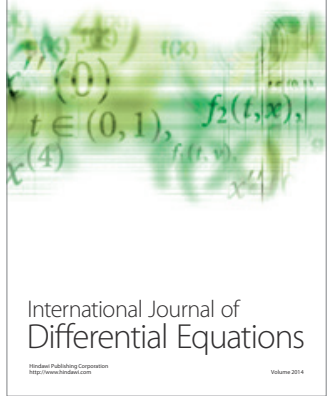
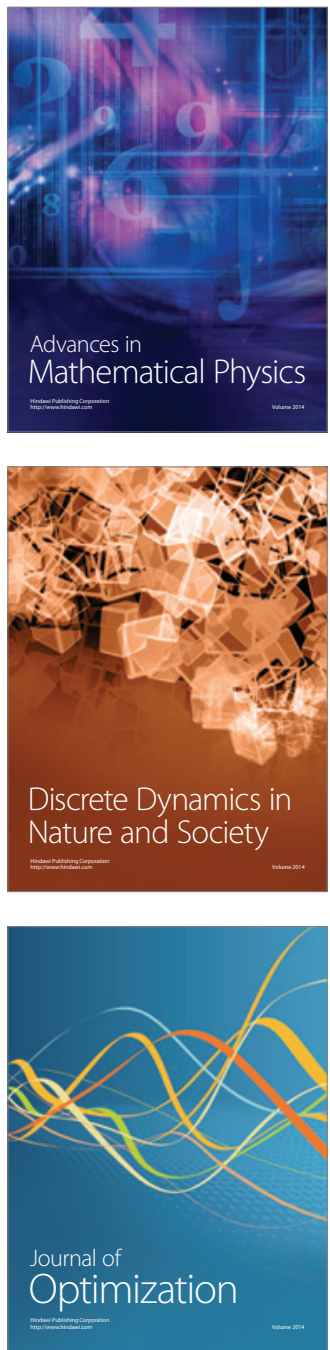